\title{
The Quantum Postulate and the Recent Development of Atomic Theory.
}

\author{
By Prof. N. BoHr, For. Mem. R.S.
}

$\mathrm{I}^{\mathrm{N}}$ connexion with the discussion of the physical interpretation of the quantum theoretical methods developed during recent years, I should like to make the following general remarks regarding the principles underlying the description of atomic phenomena, which I hope may help to harmonise the different views, apparently so divergent, concerning this subject.

\section{Quantum Postulate and Causality.}

The quantum theory is characterised by the acknowledgment of a fundamental limitation in the classical physical ideas when applied to atomic phenomena. The situation thus created is of a peculiar nature, since our interpretation of the experimental material rests essentially upon the classical concepts. Notwithstanding the difficulties which hence are involved in the formulation of the quantum theory, it seems, as we shall see, that its essence may be expressed in the so-called quantum postulate, which attributes to any atomic process an essential discontinuity, or rather individuality, completely foreign to the classical theories and symbolised by Planck's quantum of action.

This postulate implies a renunciation as regards the causal space-time co-ordination of atomic processes. Indeed, our usual description of physical phenomena is based entirely on the idea that the phenomena concerned may be observed without disturbing them appreciably. This appears, for example, clearly in the theory of relativity, which has been so fruitful for the elucidation of the classical theories. As emphasised by Einstein, every observation or measurement ultimately rests on the coincidence of two independent events at the same space-time point. Just these coincidences will not be affected by any differences which the space-time co-ordination of different observers otherwise may exhibit. Now the quantum postulate implies that any observation of atomic phenomena will involve an interaction with the agency of observation not to be neglected. Accordingly, an independent reality in the ordinary physical sense can neither be ascribed to the phenomena nor to the agencies of observation. After all, the concept of observation is in so far arbitrary as it depends upon which objects are included in the system to be observed. Ultimately every observation can of course be reduced to our sense perceptions. The circumstance, however, that in interpreting observations use has always to be made of theoretical notions, entails that for every particular case it is a question of convenience

- The content of this paper is essentially the same as that of a lecture on the present state of the quantum theory delivered on Sept. 16, 1927, at the Volta celebration in Como. For a summary of the theory just previous to the development of the new methods the reader is just previous to the development of the new methods the reader refered the a lecture dics," published in this perodid development which has taken place since has given rise to a consider able number of publications. The present paper is confined to few references to recent articles which have a special bearing on the at what point the concept of observation involving the quantum postulate with its inherent 'irrationality' is brought in.

This situation has far-reaching consequences. On one hand, the definition of the state of a physical system, as ordinarily understood, claims the elimination of all external disturbances. But in that case, according to the quantum postulate, any observation will be impossible, and, above all, the concepts of space and time lose their immediate sense. On the other hand, if in order to make observation possible we permit certain interactions with suitable agencies of measurement, not belonging to the system, an unambiguous definition of the state of the system is naturally no longer possible, and there can be no question of causality in the ordinary sense of the word. The very nature of the quantum theory thus forces us to regard the spacetime co-ordination and the claim of causality, the union of which characterises the classical theories, as complementary but exclusive features of the description, symbolising the idealisation of observation and definition respectively. Just as the relativity theory has taught us that the convenience of distinguishing sharply between space and time rests solely on the smallness of the velocities ordinarily met with compared to the velocity of light, we learn from the quantum theory that the appropriateness of our usual causal space-time description depends entirely upon the small value of the quantum of action as compared to the actions involved in ordinary sense perceptions. Indeed, in the description of atomic phenomena, the quantum postulate presents us with the task of developing a ' complementarity' theory the consistency of which can be judged only by weighing the possibilities of definition and observation.

This view is already clearly brought out by the much-discussed question of the nature of light and the ultimate constituents of matter. As regards light, its propagation in space and time is adequately expressed by the electromagnetic theory. Especially the interference phenomena in vacuo and the optical properties of material media are completely governed by the wave theory superposition principle. Nevertheless, the conservation of energy and momentum during the interaction between radiation and matter, as evident in the photoelectric and Compton effect, finds its adequate expression just in the light quantum idea put forward by Einstein. As is well known, the doubts regarding the validity of the superposition principle on one hand and of the conservation laws on the other, which were suggested by this apparent contradiction, have been definitely disproved through direct experiments. This situation would seem clearly to indicate the impossibility of a causal space-time description of the light phenomena. On one hand, in attempting to trace 
the laws of the time-spatial propagation of light according to the quantum postulate, we are confined to statistical considerations. On the other hand, the fulfilment of the claim of causality for the individual light processes, characterised by the quantum of action, entails a renunciation as regards the space-time description. Of course, there can be no question of a quite independent application of the ideas of space and time and of causality. The two views of the nature of light are rather to be considered as different attempts at an interpretation of experimental evidence in which the limitation of the classical concepts is expressed in complementary ways.

The problem of the nature of the constituents of matter presents us with an analogous situation. The individuality of the elementary electrical corpuscles is forced upon us by general evidence. Nevertheless, recent experience, above all the discovery of the selective reflection of electrons from metal crystals, requires the use of the wave theory superposition principle in accordance with the original ideas of L. de Broglie. Just as in the case of light, we have consequently in the question of the nature of matter, so far as we adhere to classical concepts, to face an inevitable dilemma, which has to be regarded as the very expression of experimental evidence. In fact, here again we are not dealing with contradictory but with complementary pictures of the phenomena, which only together offer a natural generalisation of the classical mode of description. In the discussion of these questions, it must be kept in mind that, according to the view taken above, radiation in free space as well as isolated material particles are abstractions, their properties on the quantum theory being definable and observable only through their interaction with other systems. Nevertheless, these abstractions are, as we shall see, indispensable for a description of experience in connexion with our ordinary space-time view.

The difficulties with which a causal space-time description is confronted in the quantum theory, and which have been the subject of repeated discussions, are now placed into the foreground by the recent development of the symbolic methods. An important contribution to the problem of a consistent application of these methods has been made lately by Heisenberg (Zeitschr. f. Phys., 43,$172 ;$ 1927). In particular, he has stressed the peculiar reciprocal uncertainty which affects all measurements of atomic quantities. Before we enter upon his results it will be advantageous to show how the complementary nature of the description appearing in this uncertainty is unavoidable already in an analysis of the most elementary concepts employed in interpreting experience.

\section{Quantum of Action and Kinematics.}

The fundamental contrast between the quantum of action and the classical concepts is immediately apparent from the simple formulæ which form the common foundation of the theory of light quanta and of the wave theory of material particles. If
Planck's constant be denoted by $h$, as is well known,

$$
E \tau=I \lambda=h \text {, }
$$

where $E$ and $I$ are energy and momentum respectively, $\tau$ and $\lambda$ the corresponding period of vibration and wave-length. In these formulæ the two notions of light and also of matter enter in sharp contrast. While energy and momentum are associated with the concept of particles, and hence may be characterised according to the classical point of view by definite space-time co-ordinates, the period of vibration and wave-length refer to a plane harmonic wave train of unlimited extent in space and time. Only with the aid of the superposition principle does it become possible to attain a connexion with the ordinary mode of description. Indeed, a limitation of the extent of the wavefields in space and time can always be regarded as resulting from the interference of a group of elementary harmonic waves. As shown by de Broglie (Thèse, Paris, 1924), the translational velocity of the individuals associated with the waves can be represented by just the so-called group-velocity. Let us denote a plane elementary wave by

$$
A \cos 2 \pi\left(\nu t-x \sigma_{x}-y \sigma_{y}-z \sigma_{z}+\delta\right) \text {, }
$$

where $A$ and $\delta$ are constants determining respectively the amplitude and the phase. The quantity $v=\mathbf{1} / \tau$ is the frequency, $\sigma_{x}, \sigma_{y}, \sigma_{z}$ the wave numbers in the direction of the co-ordinate axes, which may be regarded as vector components of the wave number $\sigma=1 / \lambda$ in the direction of propagation. While the wave or phase velocity is given by $v / \sigma$, the group-velocity is defined by $d v / d \sigma$. Now according to the relativity theory we have for a particle with the velocity $v$ :

$$
I=\frac{v}{c^{2}} E \text { and } v d I=d E,
$$

where $c$ denotes the velocity of light. Hence by equation (1) the phase velocity is $c^{2} / v$ and the groupvelocity $v$. The circumstance that the former is in general greater than the velocity of light empha. sises the symbolic character of these considerations. At the same time, the possibility of identifying the velocity of the particle with the group-velocity indicates the field of application of space-time pictures in the quantum theory. Here the complementary character of the description appears, since the use of wave-groups is necessarily accompanied by a lack of sharpness in the definition of period and wave-length, and hence also in the definition of the corresponding energy and momentum as given by relation (1).

Rigorously speaking, a limited wave-field can only be obtained by the superposition of a manifold of elementary waves corresponding to all values of $v$ and $\sigma_{x}, \sigma_{y}, \sigma_{z}$. But the order of magnitude of the mean difference between these values for two elementary waves in the group is given in the most favourable case by the condition

$$
\Delta t \Delta \nu=\Delta x \Delta \sigma_{x}=\Delta y \Delta \sigma_{y}=\Delta z \Delta \sigma_{z}=1,
$$

where $\Delta t, \Delta x, \Delta y, \Delta z$ denote the extension of the wave-field in time and in the directions of space corresponding to the co-ordinate axes. These 
relations-well known from the theory of optical instruments, especially from Rayleigh's investigation of the resolving power of spectral apparatus -express the condition that the wave-trains extinguish each other by interference at the space-time boundary of the wave-field. They may be regarded also as signifying that the group as a whole has no phase in the same sense as the elementary waves. From equation (1) we find thus :

$$
\Delta t \Delta E=\Delta x \Delta I_{x}=\Delta y \Delta I_{y}=\Delta z \Delta I_{z}=h \quad .
$$

as determining the highest possible accuracy in the definition of the energy and momentum of the individuals associated with the wave-field. In general, the conditions for attributing an energy and a momentum value to a wave-field by means of formula (1) are much less favourable. Even if the composition of the wave-group corresponds in the beginning to the relations (2), it will in the course of time be subject to such changes that it becomes less and less suitable for representing an individual. It is this very circumstance which gives rise to the paradoxical character of the problem of the nature of light and of material particles. The limitation in the classical concepts expressed through relation (2) is, besides, closely connected with the limited validity of classical mechanics, which in the wave theory of matter corresponds to the geometrical optics, in which the propagation of waves is depicted through 'rays.' Only in this limit can energy and momentum be unambiguously defined on the basis of space-time pictures. For a general definition of these concepts we are confined to the conservation laws, the rational formulation of which has been a fundamental problem for the symbolical methods to be mentioned below.

In the language of the relativity theory, the content of the relations (2) may be summarised in the statement that according to the quantum theory a general reciprocal relation exists between the maximum sharpness of definition of the spacetime and energy-momentum vectors associated with the individuals. This circumstance may be regarded as a simple symbolical expression for the complementary nature of the space-time description and the claims of causality. At the same time, however, the general character of this relation makes it possible to a certain extent to reconcile the conservation laws with the space-time coordination of observations, the idea of a coincidence of well-defined events in a space-time point being replaced by that of unsharply defined individuals within finite space-time regions.

This circumstance permits us to avoid the well-known paradoxes which are encountered in attempting to describe the scattering of radiation by free electrical particles as well as the collision of two such particles. According to the classical concepts, the description of the scattering requires a finite extent of the radiation in space and time, while in the change of the motion of the electron demanded by the quantum postulate one seemingly is dealing with an instantaneous effect taking place at a definite point in space. Just as in the case of radiation, however, it is impossible to define momentum and energy for an electron without considering a finite space-time region. Furthermore, an application of the conservation laws to the process implies that the accuracy of definition of the energy momentum vector is the same for the radiation and the electron. In consequence, according to relation (2), the associated space-time regions can be given the same size for both individuals in interaction.

A similar remark applies to the collision between two material particles, although the significance of the quantum postulate for this phenomenon was disregarded before the necessity of the wave concept was realised. Here this postulate does indeed represent the idea of the individuality of the particles which, transcending the space-time description, meets the claim of causality. While the physical content of the light quantum idea is wholly connected with the conservation theorems for energy and momentum, in the case of the electrical particles the electric charge has to be taken into account in this connexion. It is scarcely necessary to mention that for a more detailed description of the interaction between individuals we cannot restrict ourselves to the facts expressed by formulæ (1) and (2), but must resort to a procedure which allows us to take into account the coupling of the individuals, characterising the interaction in question, where just the importance of the electric charge appears. As we shall see, such a procedure necessitates a further departure from visualisation in the usual sense.

\section{Measurements in the Quantum Theory.}

In his investigations already mentioned on the consistency of the quantum theoretical methods, Heisenberg has given the relation (2) as an expression for the maximum precision with which the space-time co-ordinates and momentumenergy components of a particle can be measured simultaneously. His view was based on the following consideration: On one hand, the coordinates of a particle can be measured with any desired degree of accuracy by using, for example, an optical instrument, provided radiation of sufficiently short wave-length is used for illumination. According to the quantum theory, however, the scattering of radiation from the object is always connected with a finite change in momentum, which is the larger the smaller the wave-length of the radiation used. The momentum of a particle, on the other hand, can be determined with any desired degree of accuracy by measuring, for example, the Doppler effect of the scattered radiation, provided the wave-length of the radiation is so large that the effect of recoil can be neglected, but then the determination of the space co-ordinates of the particle becomes correspondingly less accurate.

The essence of this consideration is the inevitability of the quantum postulate in the estimation of the possibilities of measurement. A closer investigation of the possibilities of definition would 
still seem necessary in order to bring out the general complementary character of the description. Indeed, a discontinuous change of energy and momentum during observation could not prevent us from ascribing accurate values to the space-time co-ordinates, as well as to the momentum-energy components before and after the process. The reciprocal uncertainty which always affects the values of these quantities is, as will be clear from the preceding analysis, essentially an outcome of the limited accuracy with which changes in energy and momentum can be defined, when the wavefields used for the determination of the space-time co-ordinates of the particle are sufficiently small.

In using an optical instrument for determinations of position, it is necessary to remember that the formation of the image always requires a convergent beam of light. Denoting by $\lambda$ the wave-length of the radiation used, and by $\epsilon$ the so-called numerical aperture, that is, the sine of half the angle of convergence, the resolving power of a microscope is given by the well-known expression $\lambda / 2 \epsilon$. Even if the object is illuminated by parallel light, so that the momentum $h / \lambda$ of the incident light quantum is known both as regards magnitude and direction, the finite value of the aperture will prevent an exact knowledge of the recoil accompanying the scattering. Also, even if the momentum of the particle were accurately known before the scattering process, our knowledge of the component of momentum parallel to the focal plane after the observation would be affected by an uncertainty amounting to $2 \epsilon h / \lambda$. The product of the least inaccuracies with which the positional co-ordinate and the component of momentum in a definite direction can be ascertained is therefore just given by formula (2). One might perhaps expect that in estimating the accuracy of determining the position, not only the convergence but also the length of the wave-train has to be taken into account, because the particle could change its place during the finite time of illumination. Due to the fact, however, that the exact knowledge of the wave-length is immaterial for the above estimate, it will be realised that for any value of the aperture the wave-train can always be taken so short that a change of position of the particle during the time of observation may be neglected in comparison to the lack of sharpness inherent in the determination of position due to the finite resolving power of the microscope.

In measuring momentum with the aid of the Doppler effect-with due regard to the Compton effect - one will employ a parallel wave-train. For the accuracy, however, with which the change in wave-length of the scattered radiation can be measured the extent of the wave-train in the direction of propagation is essential. If we assume that the directions of the incident and scattered radiation are parallel and opposite respectively to the direction of the position co-ordinate and momentum component to be measured, then $c \lambda / 2 l$ can be taken as a measure of the accuracy in the determination of the velocity, where $l$ denotes the length of the wave-train. For sim- plicity, we here have regarded the velocity of light as large compared to the velocity of the particle. If $m$ represents the mass of the particle, then the uncertainty attached to the value of the momentum after observation is $c m \lambda / 2 l$. In this case the magnitude of the recoil, $2 h / \lambda$, is sufficiently well defined in order not to give rise to an appreciable uncertainty in the value of the momentum of the particle after observation. Indeed, the general theory of the Compton effect allows us to compute the momentum components in the direction of the radiation before and after the recoil from the wavelengths of the incident and scattered radiation. Even if the positional co-ordinates of the particle were accurately known in the beginning, our knowledge of the position after observation nevertheless will be affected by an uncertainty. Indeed, on account of the impossibility of attributing a definite instant to the recoil, we know the mean velocity in the direction of observation during the scattering process only with an accuracy $2 h / m \lambda$. The uncertainty in the position after observation hence is $2 h l / m c \lambda$. Here, too, the product of the inaccuracies in the measurement of position and momentum is thus given by the general formula (2).

Just as in the case of the determination of position, the time of the process of observation for the determination of momentum may be made as short as is desired if only the wavelength of the radiation used is sufficiently small. The fact that the recoil then gets larger does not, as we have seen, affect the accuracy of measurement. It should further be mentioned, that in referring to the velocity of a particle as we have here done repeatedly, the purpose has only been to obtain a connexion with the ordinary space-time description convenient in this case. As it appears already from the considerations of de Broglie mentioned above, the concept of velocity must always in the quantum theory be handled with caution. It will also be seen that an unambiguous definition of this concept is excluded by the quantum postulate. This is particularly to be remembered when comparing the results of successive observations. Indeed, the position of an individual at two given moments can be measured with any desired degree of accuracy; but if, from such measurements, we would calculate the velocity of the individual in the ordinary way, it must be clearly realised that we are dealing with an abstraction, from which no unambiguous information concerning the previous or future behaviour of the individual can be obtained.

According to the above considerations regarding the possibilities of definition of the properties of individuals, it will obviously make no difference in the discussion of the accuracy of measurements of position and momentum of a particle if collisions with other material particles are considered instead of scattering of radiation. In both cases we see that the uncertainty in question equally affects the description of the agency of measurement and of the object. In fact, this uncertainty cannot be avoided in a description of the behaviour of individuals with respect to a co-ordinate system 
fixed in the ordinary way by means of solid bodies and unperturbable clocks. The experimental devices-opening and closing of apertures, etc.are seen to permit only conclusions regarding the space-time extension of the associated wave-fields.

In tracing observations back to our sensations, once more regard has to be taken to the quantum postulate in connexion with the perception of the agency of observation, be it through its direct action upon the eye or by means of suitable auxiliaries such as photographic plates, Wilson clouds, etc. It is easily seen, however, that the resulting additional statistical element will not influence the uncertainty in the description of the object. It might even be conjectured that the arbitrariness in what is regarded as object and what as agency of observation would open up a possibility of avoiding this uncertainty altogether. In connexion with the measurement of the position of a particle, one might, for example, ask whether the momentum transmitted by the scattering could not be determined by means of the conservation theorem from a measurement of the change of momentum of the microscope-including light source and photographic plate-during the process of observation. A closer investigation shows, however, that such a measurement is impossible, if at the same time one wants to know the position of the microscope with sufficient accuracy. In fact, it follows from the experiences which have found expression in the wave theory of matter, that the position of the centre of gravity of a body and its total momentum can only be defined within the limits of reciprocal accuracy given by relation (2).

Strictly speaking, the idea of observation belongs to the causal space-time way of description. Due to the general character of relation (2), however, this idea can be consistently utilised also in the quantum theory, if only the uncertainty expressed through this relation is taken into account. As remarked by Heisenberg, one may even obtain an instructive illustration to the quantum theoretical description of atomic (microscopic) phenomena by comparing this uncertainty with the uncertainty, due to imperfect measurements, inherently contained in any observation as considered in the ordinary description of natural phenomena. He remarks on that occasion that even in the case of macroscopic phenomena we may say, in a certain sense, that they are created by repeated observations. It must not be forgotten, however, that in the classical theories any succeeding observation permits a prediction of future events with everincreasing accuracy, because it improves our knowledge of the initial state of the system. According to the quantum theory, just the impossibility of neglecting the interaction with the agency of measurement means that every observation introduces a new uncontrollable element. Indeed, it follows from the above considerations that the measurement of the positional coordinates of a particle is accompanied not only by a finite change in the dynamical variables, but also the fixation of its position means a complete rupture in the causal description of its dynamical behaviour, while the determination of its momentum always implies a gap in the knowledge of its spatial propagation. Just this situation brings out most strikingly the complementary character of the description of atomic phenomena which appears as an inevitable consequence of the contrast between the quantum postulate and the distinc. tion between object and agency of measurement, inherent in our very idea of observation.

\section{Correspondence Principle and Matrix THEORY.}

Hitherto we have only regarded certain general features of the quantum problem. The situation implies, however, that the main stress has to be laid on the formulation of the laws governing the interaction between the objects which we symbolise by the abstractions of isolated particles and radiation. Points of attack for this formulation are presented in the first place by the problem of atomic constitution. As is well known, it has been possible here, by means of an elementary use of classical concepts and in harmony with the quan. tum postulate, to throw light on essential aspects of experience. For example, the experiments regarding the excitation of spectra by electronic impacts and by radiation are adequately accounted for on the assumption of discrete stationary states and individual transition processes. This is primarily due to the circumstance that in these questions no closer description of the space-time behaviour of the processes is required.

Here the contrast with the ordinary way of description appears strikingly in the circumstance that spectral lines, which on the classical view would be ascribed to the same state of the atom, will, according to the quantum postulate, correspond to separate transition processes, between which the excited atom has a choice. Notwithstanding this contrast, however, a formal connexion with the classical ideas could be obtained in the limit, where the relative difference in the properties of neighbouring stationary states vanishes asymptotically and where in statistical applications the discontinuities may be disregarded. Through this connexion it was possible to a large extent to interpret the regularities of spectra on the basis of our ideas about the structure of the atom.

The aim of regarding the quantum theory as a rational generalisation of the classical theories led to the formulation of the so-called correspondence principle. The utilisation of this principle for the interpretation of spectroscopic results was based on a symbolical application of classical electrodynamies, in which the individual transition processes were each associated with a harmonic in the motion of the atomic particles to be expected according to ordinary mechanics. Except in the limit mentioned, where the relative difference between adjacent stationary states may be neg. lected, such a fragmentary application of the classical theories could only in certain cases lead to a strictly quantitative description of the phenomena. Especially the connexion developed by 
Ladenburg and Kramers between the classical treatment of dispersion and the statistical laws governing the radiative transition processes formulated by Einstein should be mentioned here. Although it was just Kramers' treatment of dispersion that gave important hints for the rational development of correspondence considerations, it is only through the quantum theoretical methods created in the last few years that the general aims laid down in the principle mentioned have obtained an adequate formulation.

As is known, the new development was commenced in a fundamental paper by Heisenberg, where he succeeded in emancipating himself com. pletely from the classical concept of motion by replacing from the very start the ordinary kinematical and mechanical quantities by symbols, which refer directly to the individual processes demanded by the quantum postulate. This was accomplished by substituting for the Fourier development of a classical mechanical quantity a matrix scheme, the elements of which symbolise purely harmonic vibrations and are associated with the possible transitions between stationary states. By requiring that the frequencies ascribed to the elements must always obey the combination principle for spectral lines, Heisenberg could introduce simple rules of calculation for the symbols, which permit a direct quantum theoretical transcription of the fundamental equations of classical mechanics. This ingenious attack on the dynamical problem of atomic theory proved itself from the beginning to be an exceedingly powerful and fertile method for interpreting quantitatively the experimental results. Through the work of Born and Jordan as well as of Dirac, the theory was given a formulation which can compete with classical mechanics as regards generality and consistency. Especially the element characteristic of the quantum theory, Planck's constant, appears explicitly only in the algorithms to which the symbols, the so-called matrices, are subjected. In fact, matrices, which represent canonically conjugated variables in the sense of the Hamiltonian equations, do not obey the commutative law of multiplication, but two such quantities, $q$ and $p$, have to fulfil the exchange rule

$$
p q-q p=\sqrt{-1} \frac{h}{2 \pi}
$$

Indeed, this exchange relation expresses strikingly the symbolical character of the matrix formulation of the quantum theory. The matrix theory has often been called a calculus with directly observable quantities. It must be remembered, however, that the procedure described is limited just to those problems, in which in applying the quantum postulate the space-time description may largely be disregarded, and the question of observation in the proper sense therefore placed in the background.

In pursuing further the correspondence of the quantum laws with classical mechanics, the stress placed on the statistical character of the quantum theoretical description, which is brought in by the quantum postulate, has been of fundamental importance. Here the generalisation of the symbolical method made by Dirac and Jordan represented a great progress by making possible the operation with matrices, which are not arranged according to the stationary states, but where the possible values of any set of variables may appear as indices of the matrix elements. In analogy to the interpretation considered in the original form of the theory of the 'diagonal elements' connected only with a single stationary state, as time averages of the quantity to be represented, the general transformation theory of matrices permits the representation of such averages of a mechanical quantity, in the calculation of which any set of variables characterising the 'state' of the system have given values, while the canonically conjugated variables are allowed to take all possible values. On the basis of the procedure developed by these authors and in close connexion with ideas of Born and Pauli, Heisenberg has in the paper already cited above attempted a closer analysis of the physical content of the quantum theory, especially in view of the apparently paradoxical character of the exchange relation (3). In this connexion he has formulated the relation

$$
\Delta q \Delta p \sim h \quad .
$$

as the general expression for the maximum accuracy with which two canonically conjugated variables can simultaneously be observed. In this way Heisenberg has been able to elucidate many paradoxes appearing in the application of the quantum postulate, and to a large extent to demonstrate the consistency of the symbolic method. In connexion with the complementary nature of the quantum theoretical description, we must, as already mentioned, constantly keep the possibilities of definition as well as of observation before the mind. For the discussion of just this question the method of wave mechanics developed by Schrödinger has, as we shall see, proved of great help. It permits a general application of the principle of superposition also in the problem of interaction, thus offering an immediate connexion with the above considerations concerning radiation and free particles. Below we shall return to the relation of wave mechanics to the general formulation of the quantum laws by means of the transformation theory of matrices.

\section{Wave Mechanics and Quantum Postulate.}

Already in his first considerations concerning the wave theory of material particles, de Broglie pointed out that the stationary states of an atom may be visualised as an interference effect of the phase wave associated with a bound electron. It is true that this point of view at first did not, as regards quantitative results, lead beyond the earlier methods of quantum theory, to the development of which Sommerfeld has contributed so essentially. Schrödinger, however, succeeded in developing a wave - theoretical method which has opened up new aspects, and has proved to be of decisive 
importance for the great progress in atomic physics during the last years. Indeed, the proper vibrations of the Schrödinger wave equation have been found to furnish a representation of the stationary states of an atom meeting all requirements. The energy of each state is connected with the corresponding period of vibration according to the general quantum relation (1). Furthermore, the number of nodes in the various characteristic vibrations gives a simple interpretation to the concept of quantum number which was already known from the older methods, but at first did not seem to appear in the matrix formulation. In addition, Schrödinger could associate with the solutions of the wave equation a continuous distribution of charge and current, which, if applied to a characteristic vibration, represents the electrostatic and magnetic properties of an atom in the corresponding stationary state. Similarly, the superposition of two characteristic solutions corresponds to a continuous vibrating distribution of electrical charge, which on classical electrodynamics would give rise to an emission of radiation, illustrating instructively the consequences of the quantum postulate and the correspondence requirement regarding the transition process between two stationary states formulated in matrix mechanics. Another application of the method of Schrödinger, important for the further development, has been made by Born in his investigation of the problem of collisions between atoms and free electric particles. In this connexion he succeeded in obtaining a statistical interpretation of the wave functions, allowing a calculation of the probability of the individual transition processes required by the quantum postulate. This includes a wavemechanical formulation of the adiabatic principle of Ehrenfest, the fertility of which appears strikingly in the promising investigations of Hund on the problem of formation of molecules.

In view of these results, Schrödinger has expressed the hope that the development of the wave theory will eventually remove the irrational element expressed by the quantum postulate and open the way for a complete description of atomic phenomena along the line of the classical theories. In support of this view, Schrödinger, in a recent paper (Ann.d. Phys., 83, p. 956; 1927), emphasises the fact that the discontinuous exchange of energy between atoms required by the quantum postulate, from the point of view of the wave theory, is replaced by a simple resonance phenomenon. In particular, the idea of individual stationary states would be an illusion and its applicability only an illustration of the resonance mentioned. It must be kept in mind, however, that just in the resonance problem mentioned we are concerned with a closed system which, according to the view presented here, is not accessible to observation. In fact, wave mechanics just as the matrix theory on this view represents a symbolic transcription of the problem of motion of classical mechanics adapted to the requirements of quantum theory and only to be interpreted by an explicit use of the quantum postulate. Indeed, the two formulations of the interaction problem might be said to be complementary in the same sense as the wave and particle idea in the description of the free individuals. The apparent contrast in the utilisation of the energy concept in the two theories is just connected with this difference in the startingpoint.

The fundamental difficulties opposing a spacetime description of a system of particles in interaction appear at once from the inevitability of the superposition principle in the description of the behaviour of individual particles. Already for a free particle the knowledge of energy and momentum excludes, as we have seen, the exact knowledge of its space-time co-ordinates. This implies that an immediate utilisation of the concept of energy in connexion with the classical idea of the potential energy of the system is excluded. In the Schrödinger wave equation these difficulties are avoided by replacing the classical expression of the Hamiltonian by a differential operator by means of the relation

$$
p=\sqrt{-1} \frac{h}{2 \pi} \frac{\delta}{\delta q}, \quad \text {. }
$$

where $p$ denotes a generalised component of momentum and $q$ the canonically conjugated variable. Hereby the negative value of the energy is regarded as conjugated to the time. So far, in the wave equation, time and space as well as energy and momentum are utilised in a purely formal way.

The symbolical character of Schrödinger's method appears not only from the circumstance that its simplicity, similarly to that of the matrix theory, depends essentially upon the use of imaginary arithmetic quantities. But above all there can be no question of an immediate connexion with our ordinary conceptions because the 'geometrical' problem represented by the wave equation is associated with the so-called co-ordinate space, the number of dimensions of which is equal to the number of degrees of freedom of the system, and hence in general greater than the number of dimensions of ordinary space. Further, Schrödinger's formulation of the interaction problem, just as the formulation offered by matrix theory, involves a neglect of the finite velocity of propagation of the forces claimed by relativity theory.

On the whole, it would scarcely seem justifiable, in the case of the interaction problem, to demand a visualisation by means of ordinary space-time pictures. In fact, all our knowledge concerning the internal properties of atoms is derived from experiments on their radiation or collision reactions, such that the interpretation of experimental facts ultimately depends on the abstractions of radiation in free space, and free material particles. Hence, our whole space-time view of physical phenomena, as well as the definition of energy and momentum, depends ultimately upon these abstractions. In judging the applications of these auxiliary ideas we should only demand inner consistency, in which connexion special regard has to be paid to the possibilities of definition and observation. 
In the characteristic vibrations of Schrödinger's wave equation we have, as mentioned, an adequate representation of the stationary states of an atom allowing an unambiguous definition of the energy of the system by means of the general quantum relation (I). This entails, however, that in the interpretation of observations, a fundamental renunciation regarding the space-time description is unavoidable. In fact, the consistent application of the concept of stationary states excludes, as we shall see, any specification regarding the behaviour of the separate particles in the atom. In problems where a description of this behaviour is essential, we are bound to use the general solution of the wave equation which is obtained by superposition of characteristic solutions. We meet here with a complementarity of the possibilities of definition quite analogous to that which we have considered earlier in connexion with the properties of light and free material particles. Thus, while the definition of energy and momentum of individuals is attached to the idea of a harmonic elementary wave, every space-time feature of the description of phenomena is, as we have seen, based on a consideration of the interferences taking place inside a group of such elementary waves. Also in the present case the agreement between the possibilities of observation and those of definition can be directly shown.

According to the quantum postulate any observation regarding the behaviour of the electron in the atom will be accompanied by a change in the state of the atom. As stressed by Heisenberg, this change will, in the case of atoms in stationary states of low quantum number, consist in general in the ejection of the electron from the atom. A description of the 'orbit' of the electron in the atom with the aid of subsequent observations is hence impossible in such a case. This is connected with the circumstance that from characteristic vibrations with only a few nodes no wave packages can be built up which would even approximately represent the 'motion' of a particle. The complementary nature of the description, however, appears particularly in that the use of observations concerning the behaviour of particles in the atom rests on the possibility of neglecting, during the process of observation, the interaction between the particles, thus regarding them as free. This requires, however, that the duration of the process is short compared with the natural periods of the atom, which again means that the uncertainty in the knowledge of the energy transferred in the process is large compared to the energy differences between neighbouring stationary states.

In judging the possibilities of observation it must, on the whole, be kept in mind that the wave mechanical solutions can be visualised only in so far as they can be described with the aid of the concept of free particles. Here the difference between classical mechanics and the quantum theoretical treatment of the problem of interaction appears most strikingly. In the former such a restriction is unnecessary, because the "particles" are here endowed with an immediate 'reality,' independently of their being free or bound. This situation is particularly important in connexion with the consistent utilisation of Schrödinger's electric density as a measure of the probability for electrons being present within given space regions of the atom. Remembering the restriction mentioned, this interpretation is seen to be a simple consequence of the assumption that the probability of the presence of a free electron is expressed by the electric density associated with the wave-field in a similar way to that by which the probability of the presence of a light quantum is given by the energy density of the radiation.

As already mentioned, the means for a general consistent utilisation of the classical concepts in the quantum theory have been created through the transformation theory of Dirac and Jordan, by the aid of which Heisenberg has formulated his general uncertainty relation (4). In this theory also the Schrödinger wave equation has obtained an instructive application. In fact, the characteristic solutions of this equation appear as auxiliary functions which define a transformation from matrices with indices representing the energy values of the system to other matrices, the indices of which are the possible values of the space coordinates. It is also of interest in this connexion to mention that Jordan and Klein (Zeitsch. $f$. Phys., 45, 751 ; 1927) have recently arrived at the formulation of the problem of interaction expressed by the Schrödinger wave equation, taking as starting-point the wave representation of individual particles and applying a symbolic method closely related to the deep-going treatment of the radiation problem developed by Dirac from the point of view of the matrix theory, to which we shall return below.

\section{Reality of Stationary States.}

In the conception of stationary states we are, as mentioned, concerned with a characteristic application of the quantum postulate. By its very nature this conception means a complete renunciation as regards a time description. From the point of view taken here, just this renunciation forms the necessary condition for an unambiguous definition of the energy of the atom. Moreover, the conception of a stationary state involves, strictly speaking, the exclusion of all interactions with individuals not belonging to the system. The fact that such a closed system is associated with a particular energy value may be considered as an immediate expression for the claim of causality contained in the theorem of conservation of energy. This circumstance justifies the assumption of the supra-mechanical stability of the stationary states, according to which the atom, before as well as after an external influence, always will be found in a well-defined state, and which forms the basis for the use of the quantum postulate in problems concerning atomic structure.

In a judgment of the well-known paradoxes which this assumption entails for the description of collision and radiation reactions, it is essential to consider the limitations of the possibilities of 
definition of the reacting free individuals, which is expressed by relation (2). In fact, if the definition of the energy of the reacting individuals is to be accurate to such a degree as to entitle us to speak of conservation of energy during the reaction, it is necessary, according to this relation, to coordinate to the reaction a time interval long compared to the vibration period associated with the transition process, and connected with the energy difference between the stationary states according to relation (1). This is particularly to be remembered when considering the passage of swiftly moving particles through an atom. According to the ordinary kinematics, the effective duration of such a passage would be very small as compared with the natural periods of the atom, and it seemed impossible to reconcile the principle of conservation of energy with the assumption of the stability of stationary states (cf. Zeits. $f$. Phys., 34, $142 ; 1925)$. In the wave representation, however, the time of reaction is immediately connected with the accuracy of the knowledge of the energy of the colliding particle, and hence there can never be the possibility of a contradiction with the law of conservation. In connexion with the discussion of paradoxes of the kind mentioned, Campbell (Phil. Mag., i. 1106 ; 1926) suggested the view that the conception of time itself may be essentially statistical in nature. From the view advanced here, according to which the foundation of space-time description is offered by the abstraction of free individuals, a fundamental distinction between time and space, however, would seem to be excluded by the relativity requirement. The singular position of the time in problems concerned with stationary states is, as we have seen, due to the special nature of such problems.

The application of the conception of stationary states demands that in any observation, say by means of collision or radiation reactions, permitting a distinction between different stationary states, we are entitled to disregard the previous history of the atom. The fact that the symbolical quantum theory methods ascribe a particular phase to each stationary state the value of which depends upon the previous history of the atom, would for the first moment seem to contradict the very idea of stationary states. As soon as we are really concerned with a time problem, however, the consideration of a strictly closed system is excluded. The use of simply harmonic proper vibrations in the interpretation of observations means, therefore, only a suitable idealisation which in a more rigorous discussion must always be replaced by a group of harmonic vibrations, distributed over a finite frequency interval. Now, as already mentioned, it is a general consequence of the superposition principle that it has no sense to co-ordinate a phase value to the group as a whole, in the same manner as may be done for each elementary wave constituting the group.

This inobservability of the phase, well known from the theory of optical instruments, is brought out in a particularly simple manner in a discussion of the Stern-Gerlach experiment, so important for the investigation of the properties of single atoms. As pointed out by Heisenberg, atoms with different orientation in the field may only be separated if the deviation of the beam is larger than the diffraction at the slit of the de Broglie waves representing the translational motion of the atoms. This condition means, as a simple calculation shows, that the product of the time of passage of the atom through the field, and the uncertainty due to the finite width of the beam of its energy in the field, is at least equal to the quantum of action. This result was considered by Heisenberg as a support of relation (2) as regards the reciprocal uncertainties of energy and time values. It would seem, however, that here we are not simply dealing with a measurement of the energy of the atom at a given time. But since the period of the proper vibrations of the atom in the field is connected with the total energy by relation (1), we realise that the condition for separability mentioned just means the loss of the phase. This circumstance removes also the apparent contradictions, arising in certain problems concerning the coherence of resonance radiation, which have been discussed frequently, and were also considered by Heisenberg.

To consider an atom as a closed system, as we have done above, means to neglect the spontaneous emission of radiation which even in the absence of external influences puts an upper limit to the lifetime of the stationary states. The fact that this neglect is justified in many applications is connected with the circumstance that the coupling between the atom and the radiation field, which is to be expected on classical electrodynamics, is in general very small compared to the coupling between the particles in the atom. It is, in fact, possible in a description of the state of an atom to a considerable extent to neglect the reaction of radiation, thus disregarding the unsharpness in the energy values connected with the lifetime of the stationary states according to relation (2) (cf. Proc. Camb. Phil. Soc., 1924 (Supplement), or Zeits. f. Phys., 13, 117; 1923). This is the reason why it is possible to draw conclusions concerning the properties of radiation by using classical electrodynamics.

The treatment of the radiation problem by the new quantum theoretical methods meant to begin with just a quantitative formulation of this correspondence consideration. This was the very startingpoint of the original considerations of Heisenberg. It may also be mentioned that an instructive analysis of Schrödinger's treatment of the radiation phenomena from the point of view of the correspondence principle has been recently given by Klein (Zeits. f. Phys., 41, 707; 1927). In the more rigorous form of the theory developed by Dirac (Proc. Roy. Soc., A, vol. 114, p. $243 ; 1927)$ the radiation field itself is included in the closed system under consideration. Thus it became possible in a rational way to take account of the individual character of radiation demanded by the quantum theory and to build up a dispersion theory, in which the final width of the spectral lines is taken into consideration. 
The renunciation regarding space-time pictures characterising this treatment would seem to offer a striking indication of the complementary character of the quantum theory. This is particularly to be borne in mind in judging the radical departure from the causal description of Nature met with in radiation phenomena, to which we have referred above in connexion with the excitation of spectra.

In view of the asymptotic connexion of atomic properties with classical electrodynamics, demanded by the correspondence principle, the reciprocal exclusion of the conception of stationary states and the description of the behaviour of individual particles in the atom might be regarded as a difficulty. In fact, the connexion in question means that in the limit of large quantum numbers where the relative difference between adjacent stationary states vanishes asymptotically, mechanical pictures of electronic motion may be rationally utilised. It must be emphasised, however, that this connexion cannot be regarded as a gradual transition towards classical theory in the sense that the quantum postulate would lose its significance for high quantum numbers. On the contrary, the conclusions obtained from the correspondence principle with the aid of classical pictures depend just upon the assumptions that the conception of stationary states and of individual transition processes are maintained even in this limit.

This question offers a particularly instructive example for the application of the new methods. As shown by Schrödinger (Naturwiss., 14, 664; 1926), it is possible, in the limit mentioned, by superposition of proper vibrations to construct wave groups small in comparison to the ' size' of the atom, the propagation of which indefinitely approaches the classical picture of moving material particles, if the quantum numbers are chosen sufficiently large. In the special case of a simple harmonic vibrator, he was able to show that such wave groups will keep together even for any length of time, and will oscillate to and fro in a manner corresponding to the classical picture of the motion. This circumstance Schrödinger has regarded as a support of his hope of constructing a pure wave theory without referring to the quantum postulate. As emphasised by Heisenberg, the simplicity of the case of the oscillator, however, is exceptional and intimately connected with the harmonic nature of the corresponding classical motion. Nor is there in this example any possibility for an asymptotical approach towards the problem of free particles. In general, the wave group will gradually spread over the whole region of the atom, and the 'motion' of a bound electron can only be followed during a number of periods, which is of the order of magnitude of the quantum numbers associated with the proper vibrations. This question has been more closely investigated in a recent paper by Darwin (Proc. Roy. Soc., A, vol. 117, 258 ; 1927), which contains a number of instructive examples of the behaviour of wave groups. From the viewpoint of the matrix theory a treat. ment of analogous problems has been carried out by Kennard (Zeits. f. Phys., 47, 326 ; 1927).
Here again we meet with the contrast between the wave theory superposition principle and the assumption of the individuality of particles with which we have been concerned already in the case of free particles. At the same time the asymptotical connexion with the classical theory, to which a distinction between free and bound particles is unknown, offers the possibility of a particularly simple illustration of the above considerations regarding the consistent utilisation of the concept of stationary states. As we have seen, the identification of a stationary state by means of collision or radiation reactions implies a gap in the time description, which is at least of the order of magnitude of the periods associated with transitions between stationary states. Now, in the limit of high quantum numbers these periods may be interpreted as periods of revolution. Thus we see at once that no causal connexion can be obtained between observations leading to the fixation of a stationary state and earlier observations on the behaviour of the separate particles in the atom.

Summarising, it might be said that the concepts of stationary states and individual transition processes within their proper field of application possess just as much or as little ' reality' as the very idea of individual particles. In both cases we are concerned with a demand of causality complementary to the space-time description, the adequate application of which is limited only by the restricted possibilities of definition and of observation.

\section{The Problem of the Elementary Particles.}

When due regard is taken of the complementary feature required by the quantum postulate, it seems, in fact, possible with the aid of the symbolic methods to build up a consistent theory of atomic phenomena, which may be considered as a rational generalisation of the causal space-time description of classical physics. This view does not mean, however, that classical electron theory may be regarded simply as the limiting case of a vanishing quantum of action. Indeed, the connexion of the latter theory with experience is based on assumptions which can scarcely be separated from the group of problems of the quantum theory. A hint in this direction was already given by the well-known difficulties met with in the attempts to account for the individuality of ultimate electrical particles on general mechanical and electrodynamical principles. In this respect also the general relativity theory of gravitation has not fulfilled expectations. A satisfactory solution of the problems touched upon would seem to be possible only by means of a rational quantum-theoretical transcription of the general field theory, in which the ultimate quantum of electricity has found its natural position as an expression of the feature of individuality characterising the quantum theory. Recently Klein (Zeits. f. Phys., 46, 188 ; 1927) has directed attention to the possibility of connect. ing this problem with the five-dimensional unified 
representation of electromagnetism and gravitation proposed by Kaluza. In fact, the conservation of electricity appears in this theory as an analogue to the conservation theorems for energy and momentum. Just as these concepts are complementary to the space-time description, the appropriateness of the ordinary four-dimensional description as well as its symbolical utilisation in the quantum theory would, as Klein emphasises, seem to depend essentially on the circumstance that in this description electricity always appears in well-defined units, the conjugated fifth dimension being as a consequence not open to observation.

Quite apart from these unsolved deep-going problems, the classical electron theory up to the present time has been the guide for a further development of the correspondence description in connexion with the idea first advanced by Compton that the ultimate electrical particles, besides their mass and charge, are endowed with a magnetic moment due to an angular momentum determined by the quantum of action. This assumption, introduced with striking success by Goudsmit and Uhlenbeck into the discussion of the origin of the anomalous Zeeman effect, has proved most fruitful in connexion with the new methods, as shown especially by Heisenberg and Jordan. One might say, indeed, that the hypothesis of the magnetic electron, together with the resonance problem elucidated by Heisenberg (Zeits. f. Phys., 41,$239 ; 1927$ ), which occurs in the quantumtheoretical description of the behaviour of atoms with several electrons, have brought the correspondence interpretation of the spectral laws and the periodic system to a certain degree of completion. The principles underlying this attack have even made it possible to draw conclusions regarding the properties of atomic nuclei. Thus Dennison (Proc. Roy. Soc., A, vol. 115, 483; 1927), in connexion with ideas of Heisenberg and Hund, has succeeded recently in a very interesting way in showing how the explanation of the specific heat of hydrogen, hitherto beset with difficulties, can be harmonised with the assumption that the proton is endowed with a moment of momentum of the same magnitude as that of the electron. Due to its larger mass, however, a magnetic moment much smaller than that of the electron must be associated with the proton.

The insufficiency of the methods hitherto developed as concerns the problem of the elementary particles appears in the questions just mentioned from the fact that they do not allow of an unambiguous explanation of the difference in the behaviour of the electric elementary particles and the 'individuals' symbolised through the conception of light quanta expressed in the so-called exclusion principle formulated by Pauli. In fact, we meet in this principle, so important for the problem of atomic structure as well as for the recent development of statistical theories, with one among several possibilities, each of which fulfils the correspondence requirement. Moreover, the difficulty of satisfying the relativity requirement in quantum theory appears in a particularly striking light in connexion with the problem of the magnetic electron. Indeed, it seemed not possible to bring the promising attempts made by Darwin and Pauli in generalising the new methods to cover this problem naturally, in connexion with the relativity kinematical consideration of Thomas so fundamental for the interpretation of experimental results. Quite recently, however, Dirac (Proc. of the Roy. Soc., A, 11\%, 610 ; 1928) has been able successfully to attack the problem of the magnetic electron through a new ingenious extension of the symbolical method and so to satisfy the relativity requirement without abandoning the agreement with spectral evidence. In this attack not only the imaginary complex quantities appearing in the earlier procedures are involved, but his fundamental equations themselves contain quantities of a still higher degree of complexity, that are represented by matrices.

Already the formulation of the relativity argument implies essentially the union of the spacetime co-ordination and the demand of causality characterising the classical theories. In the adaptation of the relativity requirement to the quantum postulate we must therefore be prepared to meet with a renunciation as to visualisation in the ordinary sense going still further than in the formulation of the quantum laws considered here. Indeed, we find ourselves here on the very path taken by Einstein of adapting our modes of percep. tion borrowed from the sensations to the gradually deepening knowledge of the laws of Nature. The hindrances met with on this path originate above all in the fact that, so to say, every word in the language refers to our ordinary perception. In the quantum theory we meet this difficulty at once in the question of the inevitability of the feature of irrationality characterising the quantum postulate. I hope, however, that the idea of complementarity is suited to characterise the situation, which bears a deep-going analogy to the general difficulty in the formation of human ideas, inherent in the distinction between subject and object. 